\title{
Spectral Separation for Multispectral Image Reproduction Based on Constrained Optimization Method
}

\author{
Bangyong Sun, ${ }^{1,2}$ Han Liu, ${ }^{2}$ and Shisheng Zhou ${ }^{1}$ \\ ${ }^{1}$ School of Printing and Packing Engineering, Xi'an University of Technology, Xi'an 710048, China \\ ${ }^{2} S c h o o l$ of Automation and Information Engineering, Xi'an University of Technology, Xi'an 710048, China \\ Correspondence should be addressed to Bangyong Sun; sunbangyong@xaut.edu.cn
}

Received 11 May 2014; Accepted 2 June 2014; Published 22 June 2014

Academic Editor: Qingrui Zhang

Copyright (c) 2014 Bangyong Sun et al. This is an open access article distributed under the Creative Commons Attribution License, which permits unrestricted use, distribution, and reproduction in any medium, provided the original work is properly cited.

\begin{abstract}
The constrained optimization method is employed to calculate the colorant values of the multispectral images. Because the spectral separation from the 31-dimensional spectral reflectance to low dimensional colorant values is very complex, an inverse process based on spectral Neugebauer model and constrained optimization method is performed. Firstly, the spectral Neugebauer model is applied to predict the colorants' spectral reflectance values, and it is modified by using the Yule-Nielsen $n$-value and the effective area coverages. Then, the spectral reflectance root mean square (RRMS) error is established as the objective function for the optimization method, while the colorant values are constrained to $0 \sim 1$. At last, when the nonlinear constraints and related parameters are set appropriately, the colorant values are accurately calculated for the multispectral images corresponding to the minimum RRMS errors. In the experiment, the colorant errors of the cyan, magenta and yellow inks are all below $2.5 \%$ and the average spectral error is below $5 \%$, which indicate that the precision of the spectral separation method in this paper is acceptable.
\end{abstract}

\section{Introduction}

The objective of color reproduction is to obtain the same visual perception of the original images, and it is mainly implemented based on the metameric reproduction principle $[1,2]$. However, when the illuminants or observers change, the color consistency between the original and the hard copy is hardly maintained. Thus, in many high-accuracy reproduction areas, the originals are represented as multispectral images, not the common $R G B / C M Y K$ images $[3,4]$. If the image's spectral information is reproduced correctly, the hard copy will look the same as the original under different illuminants.

Within the multispectral image reproduction workflow, after the spectral reconstruction [5-7] and gamut mapping process [8-10], the image pixel's spectral values should be precisely converted into ink values for printing, while the conversion process is often defined as spectral separation $[11,12]$. For most printers, the primary inks are cyan, magenta, yellow, and black; hence the input spectral images' reflectances are transformed to $C M Y K$ values during spectral separation.

In fact, as most of the multispectral image pixels are 31-dimensional, it is difficult to calculate the colorant values from the spectral data straightly. Most of the spectral separation processes are based on the spectral predication models and iteration methods. The spectral predication models can be used to calculate different ink combinations' spectral values. And the iteration of the separation process will stop when the image pixel's spectral matches with the predicated spectral. Several spectral predication models can be used for spectral separation, such as multiinterpolation techniques [13], spectral Neugebauer model [14, 15], YuleNielsen model [16, 17], and Kubelka-Munk model [18, 19]. Because the spectral Neugebauer model uses less sample colors and often generates acceptable accuracy, it is widely applied to the spectral separation process. However, there are many factors which influence the separation accuracy. In this paper, the spectral Neugebauer model is modified and a nonlinear optimization method is analyzed, in order to improve 
the multispectral image's spectral separation accuracy for CMYK printers.

\section{Modification of Spectral Neugebauer Model}

2.1. The Expression of Spectral Neugebauer Model. The spectral Neugebauer model is a halftone color predication model, which calculates the spectral reflectance values within the visual wavelength from printers' colorant space. In fact it is developed from Murray-Davies model which is a monochromatic model [20]. When a primary ink is printed on the paper, if the spectral reflectance of the solid and substrate are represented as $R_{\lambda, t}$ and $R_{\lambda, s}$, respectively, and the patch's dot area is given, then its reflectance values $\widehat{R}_{\lambda}$ can be predicted by using Murray-Davies model as follows:

$$
\widehat{R}_{\lambda}=a_{t} R_{\lambda, t}+\left(1-a_{t}\right) R_{\lambda, s}
$$

where $\lambda$ is the wavelength of light within $400 \mathrm{~nm}-700 \mathrm{~nm}$ and $a_{t}$ is the fractional dot area of the printed patch.

When the monochrome Murray-Davies model is extended to predict color halftones, the spectral Neugebauer model is generated. Take the cyan, magenta, and yellow colorants printing, for example; eight possible colors exist in the halftone patch which are white (bare substrate), cyan, magenta, yellow, red (magenta + yellow), green (cyan + yellow), blue (cyan + magenta), and black (cyan + magenta + yellow), respectively. These eight halftone colors are usually defined as Neugebauer primaries. When the spectral reflectance values of the primaries are measured, the halftone patch's overall reflectance can be predicated by using the spectral Neugebauer model as follows [21]:

$$
\widehat{R}_{\lambda}=\sum_{i=1}^{8} w_{i} R_{\lambda, i}
$$

where $R_{\lambda, i}$ is the $i$ th Neugebauer primary's reflectance value at full colorant coverage, and $w_{i}$ is the corresponding weighting factor calculated by the dot areas of the cyan, magenta, and yellow inks. If $c, m$, and $y$ represent the cyan, magenta, and yellow inks' area coverage, respectively, the eight Neugebauer primaries' weighting factors can be deduced using Demichel equations, and the full expression of (2) is written as follows:

$$
\begin{aligned}
\widehat{R}_{\lambda}= & (1-c)(1-m)(1-y) R_{\lambda, W}+c(1-m)(1-y) R_{\lambda, C} \\
& +(1-c) m(1-y) R_{\lambda, M}+(1-c)(1-m) y R_{\lambda, Y} \\
& +(1-c) m y R_{\lambda, R}+c(1-m) y R_{\lambda, G} \\
& +c m(1-y) R_{\lambda, B}+c m y R_{\lambda, K} .
\end{aligned}
$$

When the CMY three-color spectral Neugebauer model is extended to $C M Y K$ four colors, there are 16 Neugebauer primaries, and the weighting factors are listed in Table $1(c$, $m, y$, and $k$ represent the cyan, magenta, yellow, and black ink's area coverage).
TABLE 1: The respective fractional area coverages of the CMYK combinations.

\begin{tabular}{lcc}
\hline Index & Weighting factor & Fractional area coverage \\
\hline 1 & $w_{w}$ & $(1-c) *(1-m) *(1-y) *(1-k)$ \\
2 & $w_{c}$ & $c *(1-m) *(1-y) *(1-k)$ \\
3 & $w_{m}$ & $(1-c) * m *(1-y) *(1-k)$ \\
4 & $w_{y}$ & $(1-c) *(1-m) * y *(1-k)$ \\
5 & $w_{k}$ & $(1-c) *(1-m) *(1-y) * k$ \\
6 & $w_{c m}$ & $c * m *(1-y) *(1-k)$ \\
7 & $w_{c y}$ & $c *(1-m) * y *(1-k)$ \\
8 & $w_{m y}$ & $(1-c) * m * y *(1-k)$ \\
9 & $w_{c m y}$ & $c * m * y *(1-k)$ \\
10 & $w_{c k}$ & $c *(1-m) *(1-y) * k$ \\
11 & $w_{m k}$ & $(1-c) * m *(1-y) * k$ \\
12 & $w_{y k}$ & $c * m * y *(1-k)$ \\
13 & $w_{c m k}$ & $c *(1-m) * y * k$ \\
14 & $w_{c y k}$ & $(1-c) * m * y * k$ \\
15 & $w_{m y k}$ & $c * m * y * k$ \\
16 & $w_{c m y k}$ &
\end{tabular}

In fact, the spectral Neugebauer model is also suitable for multi-ink color systems. If $p$ colorants are included as follows:

$$
c=\left[c_{1}, \ldots, c_{j}, \ldots, c_{p}\right]^{T} \quad c_{j} \in[0,1] \text { for } j \in\{1, \ldots, p\},
$$

there will be $2^{p}$ Neugebauer primaries, and their weighting factors can be determined by the following equation [11,22]:

$$
w_{i}=\prod_{j=1}^{p} \begin{cases}c_{j} ; & \begin{array}{l}
\text { if colorant } j \text { is part of } \\
\text { the } i \text { th Neugebauer primary }
\end{array} \\
\left(1-c_{j}\right) ; & \text { else. }\end{cases}
$$

It can be concluded that the primaries' area coverages are expressed as fractions of the total area, and they satisfy the constraint $\sum_{i=1}^{2^{p}} w_{i}=1$.

Because there are only eight Neugebauer primaries within the spectral Neugebauer model for three-color printers, the predication accuracy is very limited. In order to employ more measured halftone patches as Neugebauer primaries, the cellular spectral Neugebauer model is frequently used in which the colorant space is divided into more cellular subdomains [23]. For example, when the individual ink's area coverages are partitioned into two parts with three points [0\%, $50 \%$, and $100 \%]$, the $C M Y$ colorant space will be divided into eight cellular subdomains.

2.2. The Optimization of Spectral Neugebauer Model. There are actually several reasons which decrease the predication accuracy of the spectral Neugebauer model, such as the dot gain, light scattering and penetration, and nonlinearity between the summed and the individual primaries' reflectances. For the purpose of reducing the influence of these factors, some optimization approaches should be performed. 
2.2.1. Calculating the Effective Area Coverage Values. Within the spectral Neugebauer model, the colorants' area coverages are employed to calculate the primaries' weighting factors; thus the spectral predication accuracy is highly dependent on these area coverage values, and it is significant to obtain their optimal values. For halftone color reproduction images, the change in dot diameter is the critical factor for the print image transfer, and these changes usually lead to tonal and color shifts. For a monochrome halftone patch, its spectral reflectance is usually calculated from the remitted light reflectance of solid and halftone areas as follows:

$$
a_{t}=\frac{R_{\lambda, m}-R_{\lambda, s}}{R_{\lambda, t}-R_{\lambda, s}}
$$

where $a_{t}$ is the predicted area coverage and $R_{\lambda, m}$ is the measured reflectance values of the halftone patch. Because the reflectance values change with the wavelength, the minimum reflectance value within $400 \mathrm{~nm} \sim 700 \mathrm{~nm}$ is usually used. Actually in conventional printing techniques, a widely adopted form of area coverage is deduced from the optical density values and (6) is written as

$$
a_{t}=\frac{1-10^{-D_{r}}}{1-10^{-D_{t}}}
$$

where $D_{t}$ is the optical density of solid tone, and $D_{r}$ is the halftone optical density.

When the halftone patch is measured with a densitometer, it is not the geometrical area coverage (the ratio between dots and white paper on the measuring patch), but the "optically effective area coverage." The difference between geometric and optically effective area coverage is due to the fact of light penetration and trapping. For example, as shown in Figure 1, part of the arriving light penetrates into the paper between the dots at the unprinted areas and are trapped under the dots during reflection; thus this light seems to be absorbed (as shown in Figure 1) [24].

The above phenomenon is called "light gathering," and it causes the dots to appear optically larger than they are in reality. For this reason, the optically effective area coverage consists of the geometric area coverage and the optical area gain. The optical area gain is usually defined as dot gain value increase, and it is calculated from the area coverage of the film as a master for platemaking and the tone value printed on the substrate via the printing form. The area coverage of the film (or the pixel values after color separation for Computer-toPlate techniques) is called theoretical value $a_{t}$, and the printed halftone's area coverage is the effective value $a_{\mathrm{eff}}$; thus the dot gain $Z$ is $Z=a_{\mathrm{eff}}-a_{t}$. In fact, dot gain essentially depends on the paper's surface and its absorption/ink setting behavior, the ink rheology, the blanket, printing pressure, and so on.

In order to acquire the real area coverage for the spectral Neugebauer model, all the spectral reflectance values within the visual wavelength are considered during the effective area coverage calculation. By using the least squares analysis, (6) is described into a spectral form:

$$
\begin{aligned}
a_{\mathrm{eff}}= & \left(R_{\lambda, m}-R_{\lambda, s}\right)\left(R_{\lambda, t}-R_{\lambda, s}\right)^{T} \\
& \times\left[\left(R_{\lambda, t}-R_{\lambda, s}\right)\left(R_{\lambda, t}-R_{\lambda, s}\right)^{T}\right]^{-1} .
\end{aligned}
$$

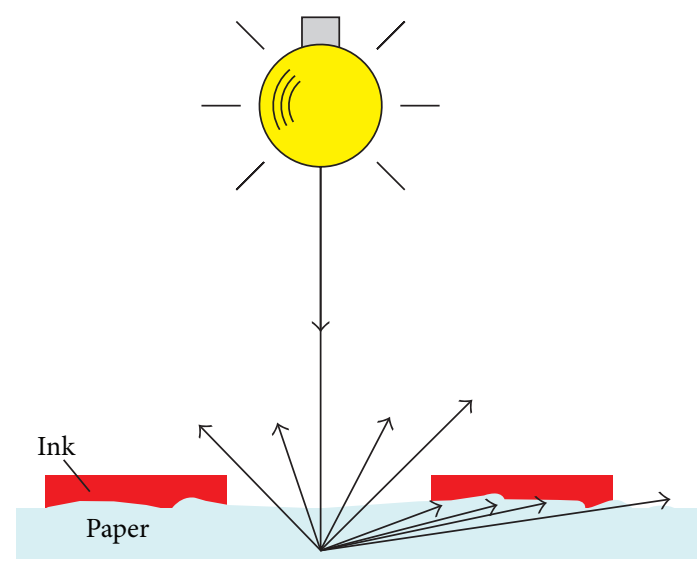

FIGURE 1: Light gathering in the area of the inked paper surface.

As we measure the reflectance from $400 \mathrm{~nm}$ to $700 \mathrm{~nm}$ with the interval of $10 \mathrm{~nm}$, all the reflectance terms above are 1 $\times 31$ row vectors, while the superscripts $T$ and -1 indicate matrix transpose and inverse, respectively. Because most of the reflectances within $400 \mathrm{~nm}-700 \mathrm{~nm}$ are involved during effective area coverage calculation in (8), it is usually more precise than (6) and (7), and we use this equation for spectral predication in the experiment.

2.2.2. Calculating the Optimal $n$-Values. As a result of light penetration and scattering effect, the relationship between measured and predicted reflectance is nonlinear for the Murray-Davies and spectral Neugebauer models. An effective solution for this problem is to add an exponent $1 / n$ to the reflectance values, which is developed by Yule and Nielsen [25]. Taking the Murray-Davies model, for example, the modified form can be described as follows:

$$
\widehat{R}_{\lambda}=\left[a_{\mathrm{eff}} R_{\lambda, t}^{1 / n}+\left(1-a_{\mathrm{eff}}\right) R_{\lambda, s}^{1 / n}\right]^{n},
$$

where $a_{\text {eff }}$ is the effective area coverage value and $n$ is a parameter accounting for light spreading in paper, and it is referred to as the Yule-Nielsen $n$-value. Generally, the modified Yule-Nielsen model is more accurate than the original Murray-Davies model. Likewise, the Yule-Nielsen model can be extended to the spectral Neugebauer model, which is called Yule-Nielsen spectral Neugebauer (YNSN) model as follows:

$$
\widehat{R}_{\lambda}=\left[\sum_{i=1}^{8} w_{i} R_{\lambda, i}^{1 / n}\right]^{n} .
$$

Because the $n$-value is not a constant, it is significant to acquire the optimal value for the specific halftone patches. Pearson [26] recommended using $n=1.7$ by testing a variety of substrates, halftone screens, and area coverages, and most of his experiment results showed that using $n=1.7$ always improved performance over using $n=1$. However, Wyble and Berns have noted that values of $n$ greater than 2 are often required for modern, high-resolution printers [27]. Arney et 


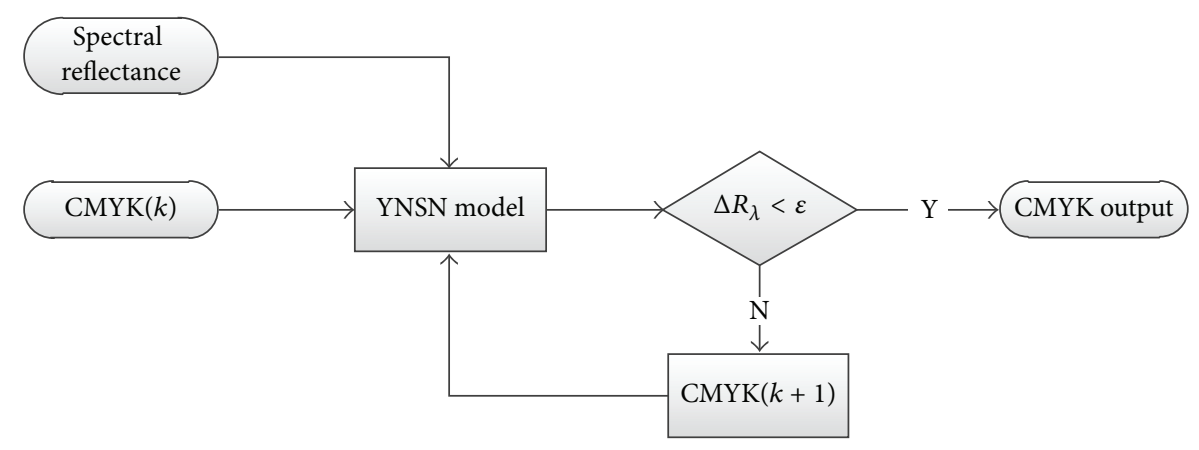

FIGURE 2: Diagram of spectral separation process based on optimization method.

al. [28] analyzed the process of light spreading in paper and approximated $n$-value as

$$
n=2-e^{-A k_{p}^{v}},
$$

where $A$ is a constant relating to dot geometry, $k_{p}$ is a constant relating to modulation transfer function, and $v$ is the halftone dot frequency in dots per millimeter. In addition, Shiraiwa and Mizuno interpreted the $n$-value in another form [29], and (9) is modified as follows based on it:

$$
\widehat{R}_{\lambda}=\left[a_{\mathrm{eff}}\left(R_{\lambda, s} T_{\lambda}^{n}\right)^{1 / n}+\left(1-a_{\mathrm{eff}}\right) R_{\lambda, s}^{1 / n}\right]^{n},
$$

where the solid ink's reflectance $R_{\lambda, t}$ is replaced by the term $R_{\lambda, t} T_{\lambda}^{n}$ and $T_{\lambda}$ is the ink transmittance. It should be noted that this model is valid only for integer values of $n$-value, and the overall effect of this is to make the predicted reflectance lower, which is consistent with the fact that the MurrayDavies model overpredicts reflectance values.

In this paper, we use a spectral-error-comparing method to find the optimal $n$-value. Firstly, the YNSN model is established with the $n$-value initialized from 1 , and then a certain number of testing patches are selected to calculate the YNSN model's spectral error. We set $n$-value ranging from 1 to 5 with the increment $\Delta n=0.1$; thus when all the spectral errors of different $n$-values are obtained and compared, the optimal value is determined corresponding to the minimal spectral error.

\section{Spectral Separation Based on Constrained Optimization Method}

For CMYK printers, it is very difficult to convert the 31dimensional spectral reflectances into the 4-dimensional CMYK colorant values. However, as the $31-\mathrm{D}$ spectral reflectance can be precisely predicted from $C M Y K$ values by spectral Neugebauer model, it is more feasible to convert the spectral separation process into the constrained optimization process based on the spectral predication model. Actually, the spectral separation is an iteration process, where the optimal CMYK values are continually searched until the predicated spectral values match well with the given spectral reflectances. Generally, the spectral separation workflow based on constrained optimization method can be described as in Figure 2.
It can be seen from the separation workflow that the objective function and the nonlinear constraints should be accurately defined for the optimization problem. Because the major purpose for printing multispectral images is to reproduce the same spectral reflectance values, the objective function is defined as the spectral reflectance error:

$$
\Delta R_{\lambda}=\left\|\widehat{R}_{\lambda, \text { CMYK }}-R_{\lambda, \text { image }}\right\|,
$$

where $\widehat{R}_{\lambda, C M Y K}$ is predicated reflectance values using YNSN model and $R_{\lambda \text {,image }}$ is the multispectral image's reflectance values. The iteration process in Figure 2 will stop when the difference of (13) is smaller than a threshold value $\varepsilon$ predefined.

In addition, as the colorant values have the specific scale range, the nonlinear constraints can be defined as follows:

$$
0 \leq a_{\mathrm{eff}} \leq 1, \quad \sum a_{\mathrm{eff}} \leq a_{\text {limit }},
$$

where $a=\{c, m, y, k\}$, and $a_{\text {limit }}$ represents the limit of the total ink amount. Thus, the nonlinear optimization process for spectral separation is described as

$$
\begin{array}{ll}
\min & \left\|\widehat{R}_{\lambda, C M Y K}-R_{\lambda, \text { image }}\right\| \\
\text { s.t. } & a_{\text {eff }} \in[0,1], \\
& \sum a_{\text {eff }} \leq a_{\text {limit }} .
\end{array}
$$

It should be noted that the use of an optimization technique may lead to a nonoptimal solution (e.g., a local minimum will stop the iteration process) as Mahy and Delabastita presented [30]. Thus, Gerhardt and Hardeberg [11] analyzed the parameters which determined the right colorant combination obtained, including the limitations on the minimum difference between the desired value and its estimation, difference between colorant values in successive iterations, and initial guess values to start the iterative search.

\section{Experiment and Analysis}

In the experiment, the cyan, magenta, and yellow inks are used to print the multispectral images, and the three-color spectral Neugebauer model is employed for spectral predication. The halftone patches are printed with a HP digital 
TABLE 2: The colorant and spectral reflectance error for 50 testing halftone samples.

\begin{tabular}{llllrr}
\hline & $\Delta C$ & $\Delta M$ & $\Delta Y$ & $\Delta E_{c m y}$ & RRMS \\
\hline Avg. (error) & 1.43 & 2.26 & 2.21 & 3.85 & 0.0047 \\
Std. (error) & 0.98 & 1.54 & 2.00 & 2.13 & 0.0030 \\
Max. (error) & 4.54 & 6.07 & 7.23 & 9.86 & 0.0128 \\
\hline
\end{tabular}

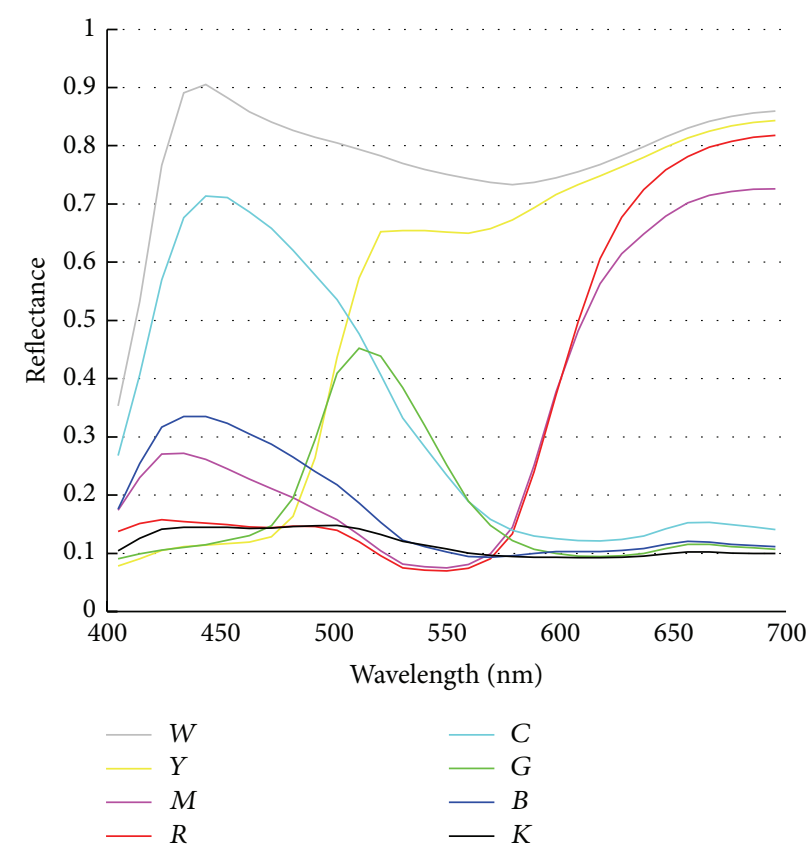

Figure 3: Spectral reflectance data of eight Neugebauer primaries.

printer, and the spectral reflectance values are measured with an $X$-Rite 530 spectrophotometer with geometry $\left(d: 10^{\circ}\right)$ under a D65 illuminant. Take the eight Neugebauer primaries of $C M Y$ space, for example, the spectral reflectances within $400 \mathrm{~nm}-700 \mathrm{~nm}$ are illustrated as in Figure 3.

Besides, 50 testing halftone patches are selected to test the spectral separation method in the paper. Firstly, the testing patches are printed with the defined ink values $C_{1} M_{1} Y_{1}$, and then the spectral reflectance values $R_{\lambda, 1}$ are measured. Secondly, by using the spectral separation method described in Sections 2 and 3, new ink values $C_{2} M_{2} Y_{2}$ are calculated from the measured spectral reflectance $R_{\lambda, 1}$. At last, when these $C_{2} M_{2} Y_{2}$ values are printed and measured, new spectral reflectance values $R_{\lambda, 2}$ are obtained. The difference of $C M Y$ values between $C_{1} M_{1} Y_{1}$ and $C_{2} M_{2} Y_{2}$ and the spectral error between $R_{\lambda, 1}$ and $R_{\lambda, 2}$ are calculated to test the separation accuracy $[23,31]$. The $C M Y$ ink difference and the reflectance error are expressed as follows:

$$
\Delta E_{C M Y}=\sqrt{\Delta C^{2}+\Delta M^{2}+\Delta Y^{2}},
$$

where $\Delta C=\left|C_{1}-C_{2}\right|, \Delta M=\left|M_{1}-M_{2}\right|$, and $\Delta Y=\left|Y_{1}-Y_{2}\right|$. While for the spectral errors, the reflectance root mean square (RRMS) difference is represented as

$$
\mathrm{RRMS}=\sqrt{\frac{\sum_{\lambda}\left[R_{\lambda, 1}-R_{\lambda, 2}\right]^{2}}{N}},
$$

where $N$ is the dimensionality of spectral reflectance, $R_{\lambda, 1}$ is the input and given reflectance values, and $R_{\lambda, 2}$ is newly predicted CMY inks' reflectance values, and they are both scaled to $0 \sim 1$. The RRMS error reveals the matching degree of two spectral reflectance values, so it may favorably evaluate the precision of spectral separation method.

In Table 2 the errors in the form of $\Delta C, \Delta M, \Delta Y$, and RRMS are listed, where the Avg(Error) represents the average of the errors, Std(Error) is the standard deviation of the errors, and Max(Error) is the maximum of the errors.

From Table 2, it can be seen that the differences of ink values are about $2 \%$, and most of the spectral reflectance errors are below 5\%, which indicates that the accuracy of the spectral separation method in this paper is acceptable. For all the 50 testing halftone patches, the colorant errors of cyan, magenta, and yellow inks are shown in Figure 4.

The spectral reflectance error is depicted in Figures 5 and 6. In Figure 5, the distribution of RRMS errors for 50 halftone patches is listed, while, in Figure 6, the original and newly separated spectral reflectance values are compared, and it can be seen that these two figures match very well.

\section{Conclusions}

The spectral separation algorithm is significant for the multispectral image printing devices, which can calculate the accurate colorant values. In the traditional printing process, the 


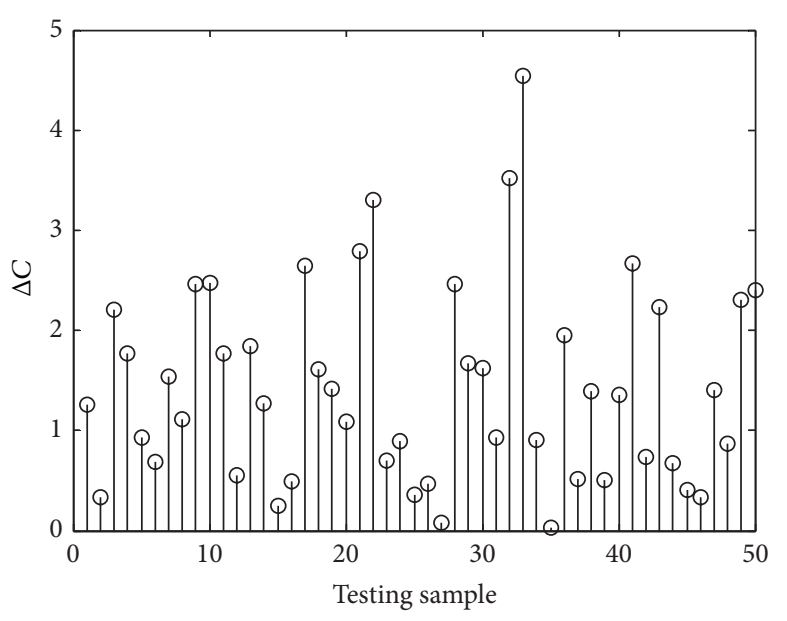

(a) The errors of cyan ink

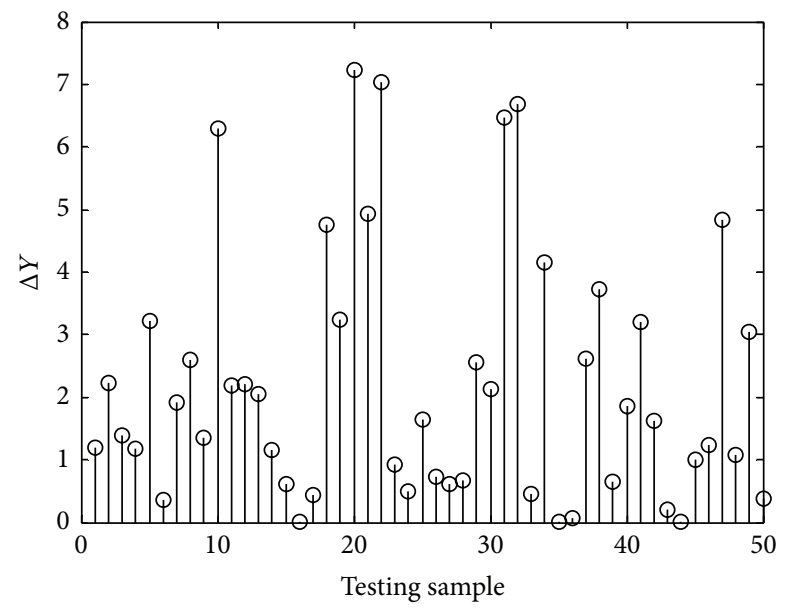

(c) The errors of yellow ink

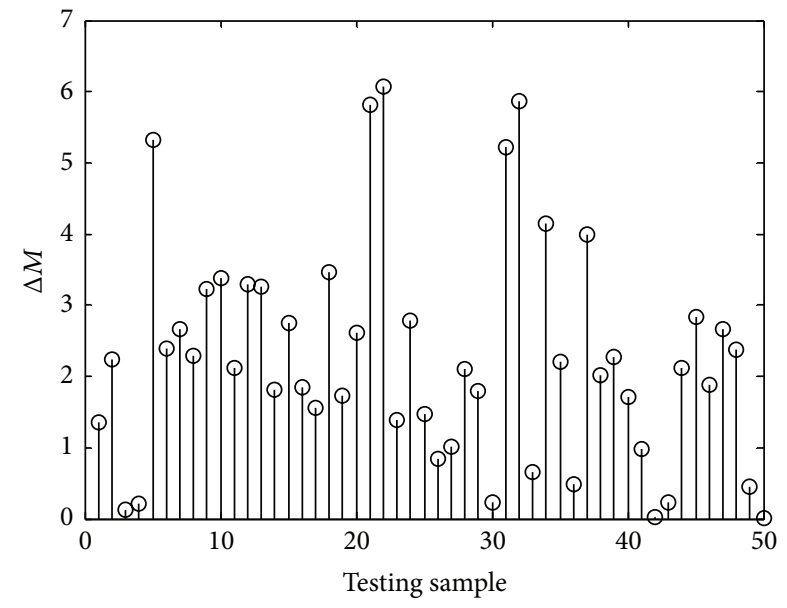

(b) The errors of magenta ink

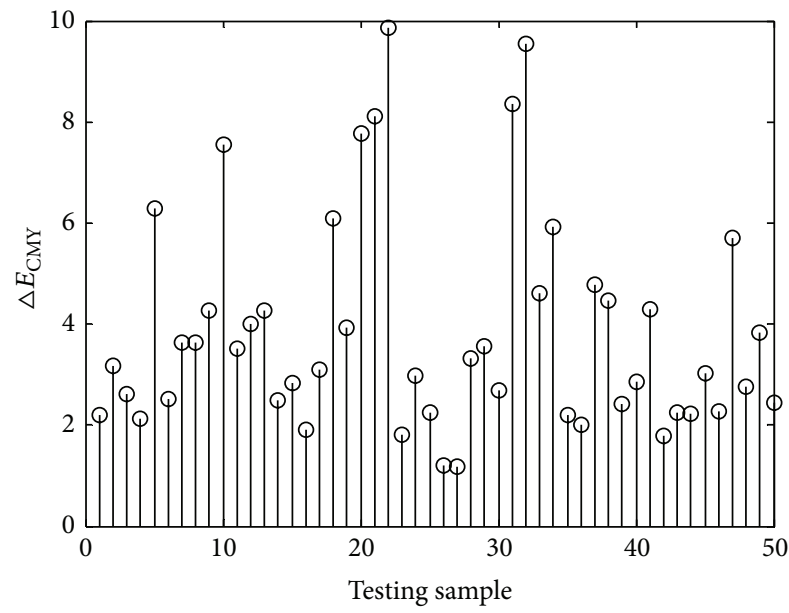

(d) The combined errors of three inks

Figure 4: Colorant errors of cyan, magenta, and yellow inks.

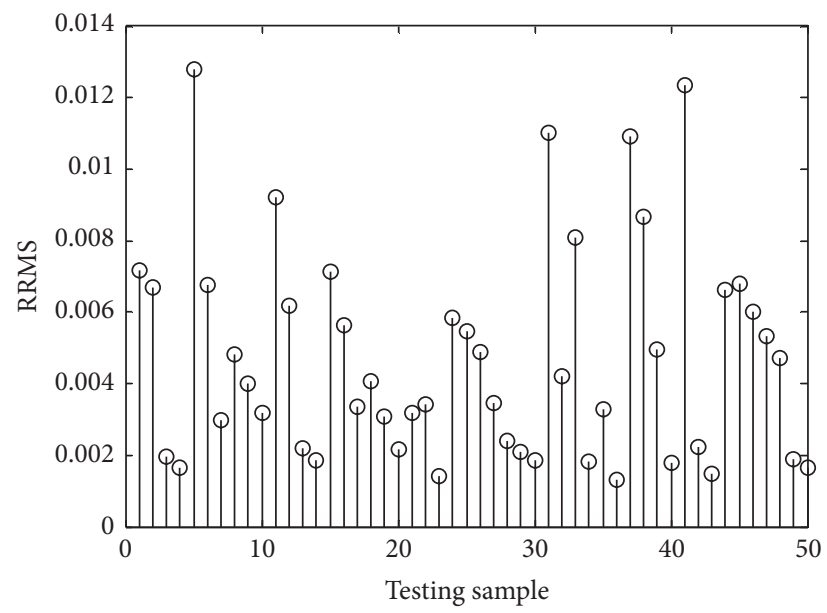

Figure 5: Spectral reflectance error of the 50 halftone patches. 


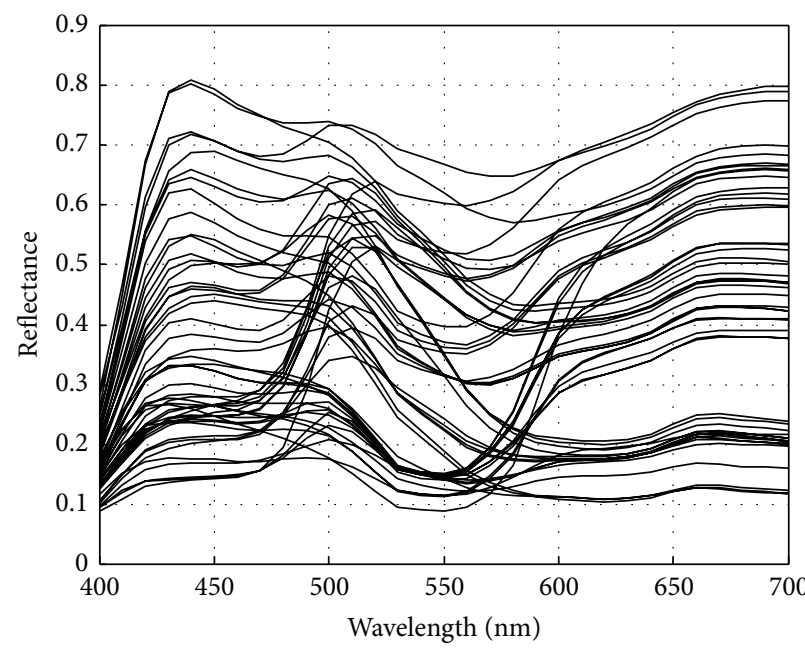

(a) Measured reflectance values

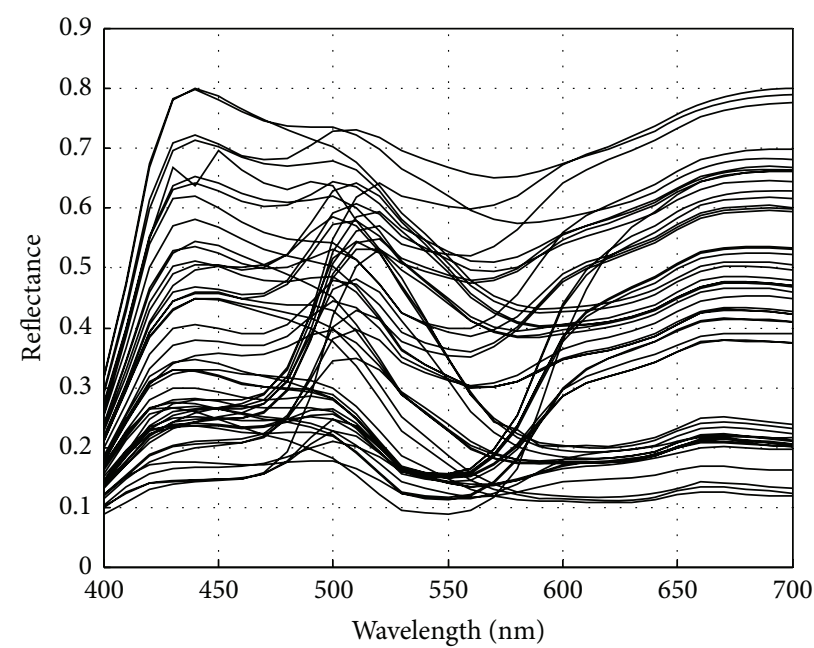

(b) New reflectance values by spectral separation

FIgURE 6: Comparison of the original and newly separated spectral values of the 50 testing samples.

multispectral images are firstly converted into the CIE color values under specific illuminant, and then the colorant values are calculated from the CIE colors. Because the connection space is the 3-dimentional CIEXYZ or CIELAB under one illuminant, the original and reproduced multispectral images cannot keep same color appearance under different observing circumstances. In this paper, the spectral separation method is employed to calculate the spectral reflectance's colorant values and the purpose of which is to generate the identical spectral values of the input multispectral images. Thus, the original and reproduced images reveal the same visual colors under different illuminants. The accuracy of the spectral separation method is evaluated in the experiment. The experiment results show that the colorants errors of three inks are about $2 \%$, and the average spectral error is below $5 \%$, which can guarantee the spectral consistence between the original and the printed multispectral images.

\section{Conflict of Interests}

The authors declare that there is no conflict of interests regarding the publication of this paper.

\section{Acknowledgments}

The authors gratefully acknowledge the financial support by research foundation of Department of Education of Shaanxi Province (no. 11JK0541), Doctor Foundation of Xi'an University of Technology (no. 104-211302), and "13115" Creative Foundation of Science and Technology (no. 2009ZDGC-06), Shaanxi Province of China.

\section{References}

[1] I. Zjakic, D. Parac-Osterman, and I. Bates, "New approach to metamerism measurement on halftone color images," Measurement, vol. 44, no. 8, pp. 1441-1447, 2011.
[2] M. Rump and R. Klein, "Spectralization: reconstructing spectra from sparse data," Computer Graphics Forum, vol. 29, no. 4, pp. 1347-1354, 2010.

[3] N. Hagen and W. Kudenov Michael, "Review of snapshot spectral imaging technologies," Optical Engineering, vol. 52, no. 9, Article ID 090901, 2013.

[4] F. Wang, A. Behrooz, M. Morris, and A. Adibi, "High-contrast subcutaneous vein detection and localization using multispectral imaging," Journal of Biomedical Optics, vol. 18, no. 5, Article ID 050504, 2013.

[5] P. Xia, Y. Shimozato, Y. Ito et al., "Improvement of color reproduction in color digital holography by using spectral estimation technique," Applied Optics, vol. 50, no. 34, pp. H177H182, 2011.

[6] P.-Y. Ren, N.-F. Liao, B.-H. Chai, W.-P. Yang, and S.-X. Li, "Spectral reflectance recovery based on multispectral imaging," Optical Technique, vol. 31, no. 3, pp. 427-433, 2005.

[7] Z. Liu, X.-X. Wan, X.-G. Huang, Q. Liu, and C. Li, “The study on spectral reflectance reconstruction based on wideband multispectral acquisition system," Spectroscopy and Spectral Analysis, vol. 33, no. 4, pp. 1076-1081, 2013.

[8] H. Li, J. Feng, W. Yang et al., "Spectral-based rendering method and Its application in multispectral color reproduction," Laser and Optoelectronics Progress, vol. 47, no. 12, Article ID 122301, 2010.

[9] P. Urban and R. S. Berns, "Paramer mismatch-based spectral gamut mapping," IEEE Transactions on Image Processing, vol. 20, no. 6, pp. 1599-1610, 2011.

[10] B. Sun, H. Liu, S. Zhou, and W. Li, "A color gamut description algorithm for liquid crystal displays in CIELAB space," The Scientific World Journal, vol. 2014, Article ID 671964, 9 pages, 2014.

[11] J. Gerhardt and J. Y. Hardeberg, "Spectral color reproduction minimizing spectral and perceptual color differences," Color Research and Application, vol. 33, no. 6, pp. 494-504, 2008.

[12] P. Urban and R.-R. Grigat, "Spectral-based color separation using linear regression iteration," Color Research and Application, vol. 31, no. 3, pp. 229-238, 2006. 
[13] D.-W. Kang, Y.-T. Kim, Y.-H. Cho, K.-H. Park, W.-H. Choe, and Y.-H. Ha, "Color decomposition method for multi-primary display using 3D-LUT in linearized LAB space," in Color Imaging X: Processing, Hardcopy, and Applications, vol. 5667 of Proceedings of SPIE, pp. 354-363, San Jose, Calif, USA, January 2005.

[14] C. Lana, M. Rotea, and D. E. Viassolo, "Robust estimation algorithm for spectral Neugebauer models," Journal of Electronic Imaging, vol. 14, no. 1, pp. 1-10, 2005.

[15] S. Xi and Y. Zhang, "Neugebauer reflectance model of frequency modulation halftone image," Optik, vol. 124, no. 15, pp. 21032105, 2013.

[16] B. Wang, H. Xu, M. Ronnier Luo, and J. Guo, "Maintaining accuracy of cellular Yule-Nielsen spectral Neugebauer models for different ink cartridges using principal component analysis," Journal of the Optical Society of America A: Optics and Image Science, and Vision, vol. 28, no. 7, pp. 1429-1435, 2011.

[17] M. Hébert and R. D. Hersch, "Yule-nielsen based recto-verso color halftone transmittance prediction model," Applied Optics, vol. 50, no. 4, pp. 519-525, 2011.

[18] C. Sandoval and D. Kim Arnold, "Deriving Kubelka-Munk theory from radiative transport," Journal of the Optical Society of America A: Optics and Image Science, and Vision, vol. 31, no. 3, pp. 628-636, 2014.

[19] L. M. Schabbach, F. Bondioli, and M. C. Fredel, "Color prediction with simplified Kubelka-Munk model in glazes containing $\mathrm{Fe}_{2} \mathrm{O}_{3}-\mathrm{ZrSiO}_{4}$ coral pink pigments," Dyes and Pigments, vol. 99, no. 3, pp. 1029-1035, 2013.

[20] J. Seymour and P. Noffke, "A universal model for halftone reflectance," in Proceedings of the Technical Association of the Graphic Arts (TAGA '12), pp. 1-40, March 2012.

[21] T. Bugnon and R. D. Hersch, "Calibrating ink spreading curves by optimal selection of tiles from printed color images," Journal of ELectronic Imaginig, vol. 21, no. 1, Article ID 013024, pp. 1-15, 2012.

[22] B. Wang, H. Xu, and L. M. Ronnier, "Color separation criteria for spectral multi-ink printer characterization," Chinese Optics Letters, vol. 10, no. 1, Article ID 013301, 2012.

[23] J. Guo, H. Xu, and L. M. Ronnier, "Novel spectral characterization method for color printer based on the cellular Neugebauer model," Chinese Optics Letters, vol. 8, no. 11, pp. 1106-1109, 2010.

[24] H. Kipphan, Handbook of Print Media, Springer, Berlin, Germany, 2001.

[25] J. A. C. Yule and W. J. Nielsen, "The penetration of light into paper and its effect on halftone reproductions," TAGA Proc, vol. 3, pp. 65-76, 1951.

[26] M. Pearson, " $n$ value for general conditions," TAGA Proc, vol. 32, pp. 415-425, 1980.

[27] D. R. Wyble and R. S. Berns, "A critical review of spectral models applied to binary color printing," Color Research and Application, vol. 25, no. 1, pp. 4-19, 2000.

[28] J. S. Arney, C. D. Arney, M. Katsube, and P. G. Engeldrum, “The impact of paper optical properties on hard copy image quality," in Proceeding of the International Conference on Digital Printing Technologies, pp. 166-167, 1996.

[29] Y. Shiraiwa and T. Mizuno, "Equation to predict colors of halftone prints considering the optical property of paper," Journal of Imaging Science and Technology, vol. 37, no. 4, pp. 385391, 1993.

[30] M. Mahy and P. Delabastita, "Inversion of the Neugebauer equations," Color Research and Application, vol. 21, no. 6, pp. 404-411, 1996.
[31] S. Shen and R. S. Berns, "Color-difference formula performance for several datasets of small color differences based on visual uncertainty," Color Research and Application, vol. 36, no. 1, pp. 15-26, 2011. 

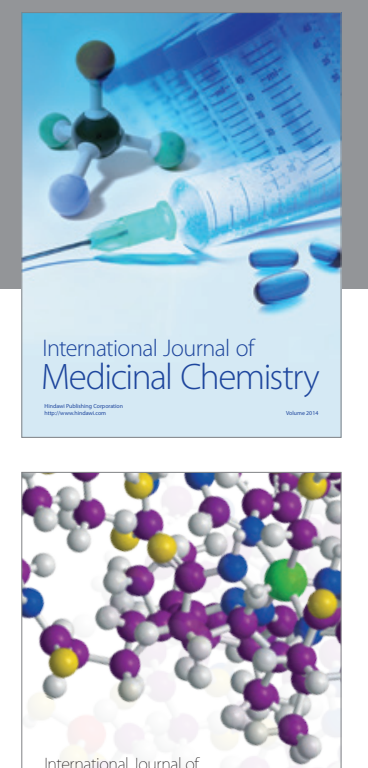

\section{Carbohydrate} Chemistry

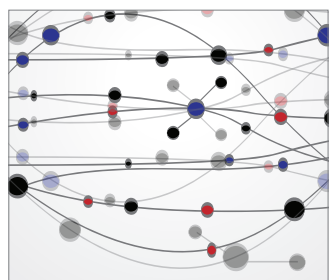

The Scientific World Journal
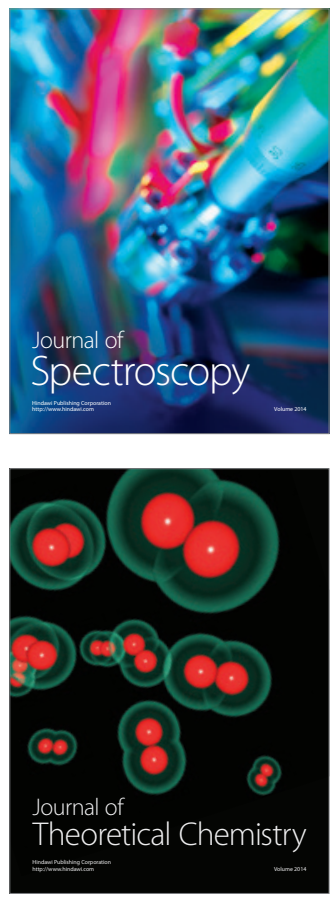
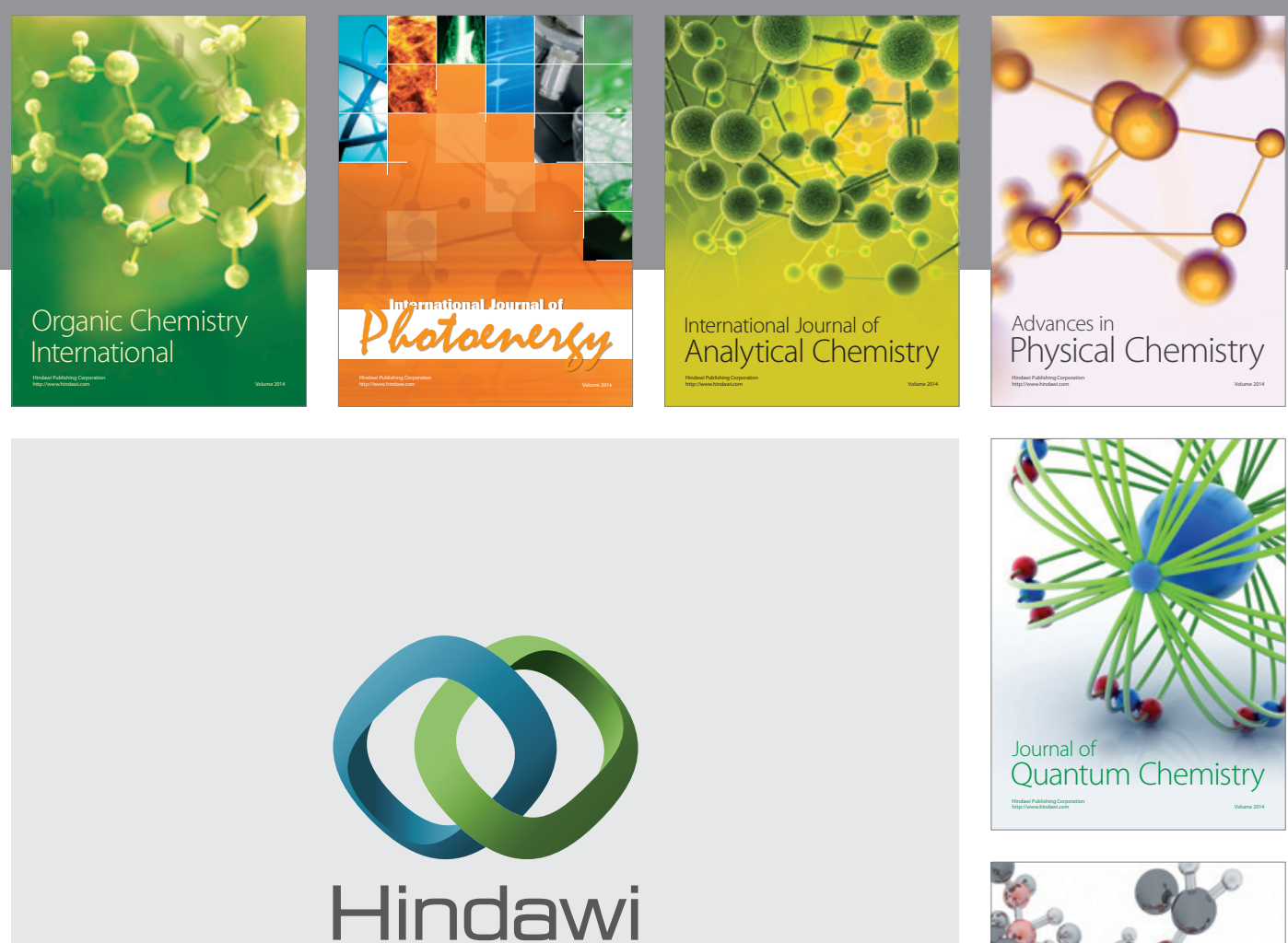

Submit your manuscripts at

http://www.hindawi.com

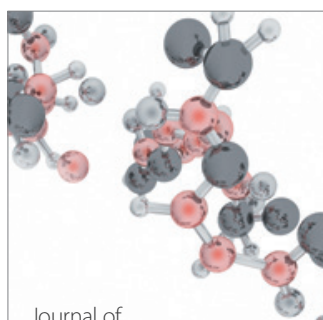

Analytical Methods

in Chemistry

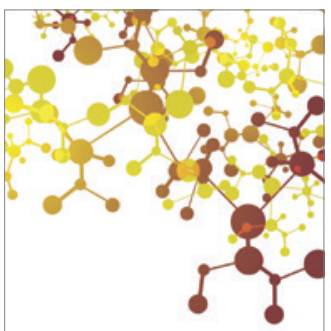

Journal of

Applied Chemistry

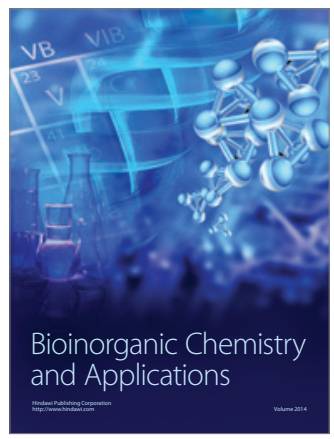

Inorganic Chemistry
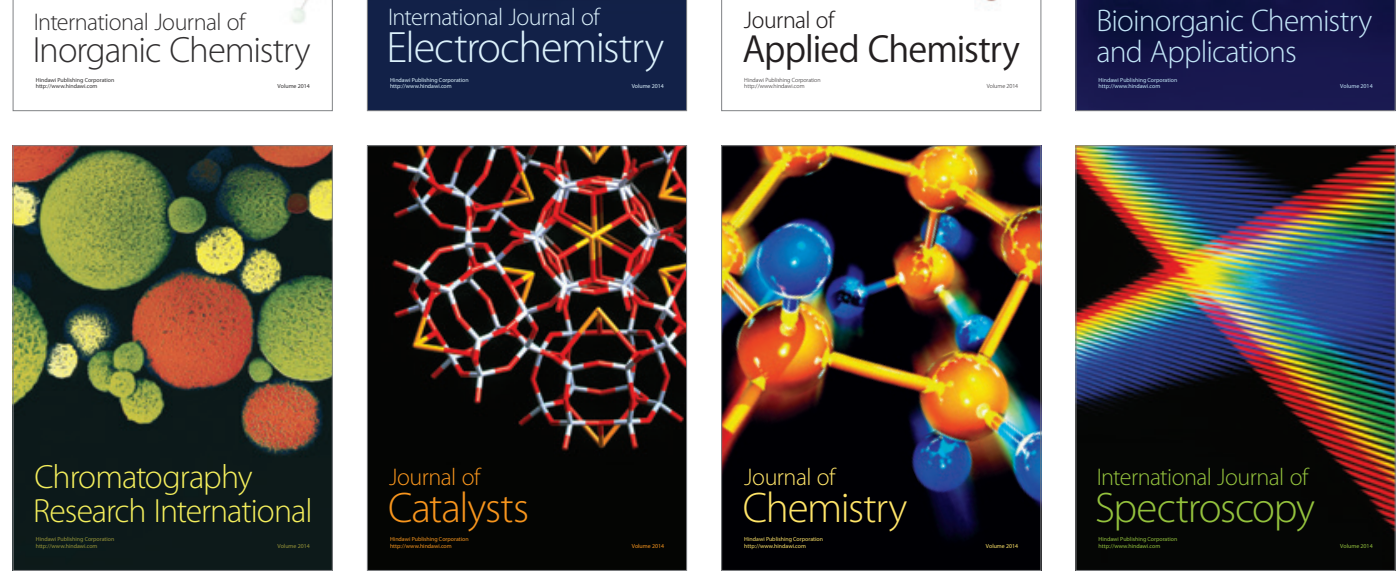